\title{
САХАРНЫЙ ДИАБЕТ И АУТОИММУННЫЙ ТИРЕОИДИТ - ЭТИОПАТОГЕНЕТИЧЕСКИЕ ВЗАИМОСВЯЗИ, СКРИНИНГ, РАННЯЯ ДИАГНОСТИКА И ЛЕЧЕНИЕ
}

\author{
1Логачев М.Ф., 1,2 Дёмина Е.С., 1,2 Комбарова А.С., ${ }^{2}$ Барская М.А. \\ ${ }^{1}$ ФГАОУ ВО РНИМУ им. Н.И. Пирогова МЗРФ \\ ²ОСП РДКБ ФГАОУ ВО РНИМУ имени Н.И. Пирогова МЗРФ \\ г. Москва, Россия
}

\begin{abstract}
Сахарный диабет 1 типа (СД-1) и аутоммунный лимфоцитарный тиреоидит (АИТ) это две из наиболее распространенных хронических болезней эндокринной системы. Поздняя диагностика АИТ на фоне СД-1 приводит к ухудшению компенсации пациентов, особенно на этапе развития у них гипотиреоза.
\end{abstract}

Целью нашей работы явилась оптимизация клинико-лабораторного скрининга для ранней диагностики АИТ и субклинического гипотиреоза при СД-1 с сопутствующими метаболическими нарушениями. Под нашим наблюдением с 2019 года по 2021 год в отделении ОСП РДКБ ФГАОУ ВО РНИМУ имени Н.И. Пирогова находилось 100 детей и подростков с СД-1 в возрасте от 3 до 17лет. Мальчиков 30, девочек 70.

Манифестация СД -1 приходилась на период от 8 месяцев до 13,5 лет с наибольшей частотой выявления в возрасте от 3 до 9 лет. Дебют признаков АИТ приходился на возраст от 3 до 16 лет. Наибольшая частота выявления АИТ отмечена в возрасте от 8 до 15 лет. Переход из фазы эутиреоза в гипотиреоз прослеживался у 11 детей (11\%), из которых 3 мальчика (18\%), 9 девочек (82\%), в период от 2 месяцев до 5 лет 8 месяцев от начала выявления АИТ . Уровень гликированного гемоглобина при этом у 3 детей (девочек) (27,3\%) был до 7\%, у остальных детей укладывался в границы от 7,1\% до 9,2\%. У 4 детей (4\%) из анализируемой группы выявлена фаза гипертиреоза в возрасте от 6,8 лет до 12,3 лет, уровень гликированного гемоглобина при выявлении данной фазы от 5,5\% до 8,5\%. У 16 человек (16\%) прослеживается фаза гипотиреоза АИТ из которых 5 мальчиков $(31,25 \%)$, у 4 человек (25\%) из низ АИТ выявлен одновременно с СД -1 . У остальных проявления фазы гипотиреоза появились в период от 3 месяцев до 7,3 лет от начала манифестации СД -1. Уровень гликированного гемоглобина при дебюте фазы гипотиреоза АИТ у 5 детей (31\%) до 7\%, у остальных колебался от 7,2\% до 10,4\%. Фаза эутиреоза АИТ наблюдалась у 61 ребенка (61\%). На терапии находилось 35 (35 \%) наблюдавшихся нами детей и подростков, что позволило значительно улучшить степень компенсации.

Таким образом, АИТ является одной из часто развивающихся при СД-1 болезней, которая не всегда достаточно рано диагностируется. Использование оптимизированного клинико-лабораторного скрининга позволяет нормализовать метаболические процессы в организме, уровень гликемии, что приводит к улучшению состояния пациентов с сахарным диабетом и значительно снизить риск развития у них осложнений. 